\title{
Orderly Disorder in modern Physics
}

\author{
S. Davood Sadatian* \\ Department of Physics, Faculty of Basic Sciences \\ University of Neyshabur, P. O. Box 9318713331, Neyshabur, Iran \\ *E-mail address: sd-sadatian@um.ac.ir
}

\begin{abstract}
Fractal structures in various subjects are taken into consideration. In this article, we study fractal structures in physics which could be found or might be existent. Basically, fractals are important because they CHANGE the most basic ways we analyze and understand physics and experimental data.
\end{abstract}

Keywords: Chaos Theory; Quantum Gravity; Symmetry Breaking; Fractal Mathematics

\section{INTRODUCTION}

Fractal geometry is composed of large components with each component in a certain proportion. In other words, Fractal structure is like each component of the straw. Fractals are shapes unlike Euclidean geometric shapes that are not organized in any way. First of all irregular shapes, and secondly, the disorder are identical in all aspects of the fractal object seen somehow identical. Since the minimal other Self-Similarity fractals, as an important tool in computer graphics, were mentioned, the emergence of this new concept will play the largest role in video compression. The fractal dimension is a fractional number called when a fractal dimension is 1.2. This means that the line is more complex than a simple page. It comes after a series of logarithmic calculation formulas. However, when you look around you can be the smallest natural elements in the creation of objects, for example, which are a form of fractal geometry, fractal characteristics raised in the model. A lot of time trying to get into this huge range that cannot be raised in a limited article. Therefore, according to the form of the fractal geometry and choose to ask[1].

In other hand, Chaos Theory (Chaos) is a mathematical concept that defines it may not be very accurate, but it can be considered a kind of randomness and uncertainty, the uncertainty because of internal reasons is that the disorder does not occur due to external disturbances, it happens because it is a behavior disorder, is irregular and unpredictable. The theory is that in the area of science, mathematics, behavioral science, management, sociology, etc., causing a change in the type of human vision is to solve unforeseen problems. Chaos Theory is the key concept in chaos, or confusion existent[2].

Finally in physics, irregularities in the laboratory and in the real world can be found in abundance. For the first time in 1898 Hdamard during the study based systems frictionless glide particles on surfaces with constant curvature of the sensitivity to the initial condition of the realized system. With respect to the initial condition, the laws of force and movement of Newton and Kepler's laws. Further studies on the theory of non-linear dynamical systems by 
scientists associated with the disorder under the Birkhoff Kolmogorov, Stephen Smale, Littlewood, Cartwright was that all studies except Smale was affected by physical problems and the manual calculations, but the computer is simply impossible to perform calculations $\mathrm{Da} \mathrm{Da}$ [3]. Possible physical symmetry breaking in some cases. The formalism of quantum gravity has opened a new window on the realm of physics. Basically, every phenomenon in the world of order and disorder in which there may be irregularities in any order. But there lies a profound change in our view of the irregularities in order to change.

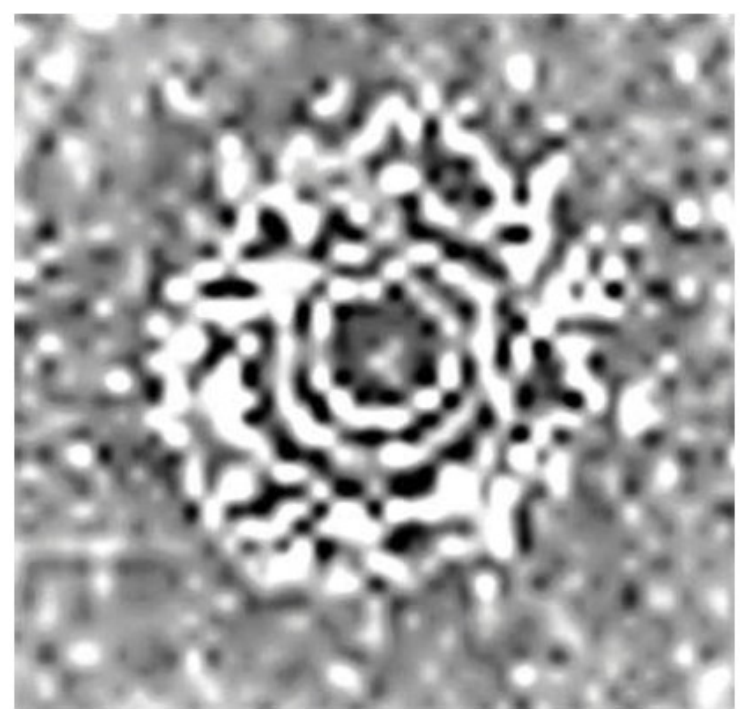

Figure 1. Ground state atom.

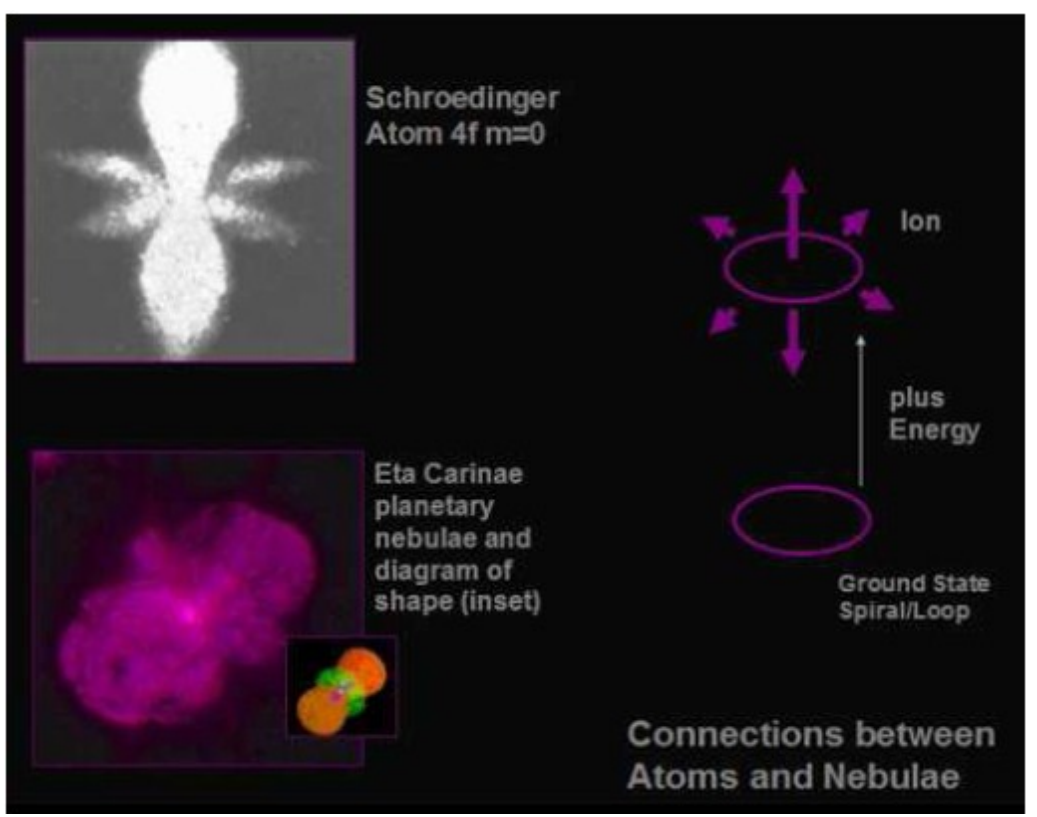

Figure 2. The resemblance of certain Schroedinger atomic shapes to planetary nebulae hints that atoms are tiny members of the postulated spiral hierarchy. Hence ejections may be expected in atoms as in galaxies, nebulae etc. 


\section{PHENOMENOLOGY}

In this section we consider fractal concept in a major physics subject. It is interesting to know to what extent fractal concepts in physics are significant. The mystery, perhaps the ultimate mystery. This is the mind / body problem and the mystery of consciousness is complex. However, only very rarely we take time to reflect upon the nature of the scientists. Dynamic concept, relying primarily on the concept of time. Time is assumed to flow in one direction, leading to a series of events that occur one after another. The conventional view is in both science and philosophy. It is not clear, however, that this is easy, since the current is entirely accurate. Good reasons to show the time as there is a spiral rather than linear. In the following we plan view of the spiral of time, from the perspective of both physics and phenomenology. Here we are in the nature of time from two points of view: Physics and Phenomenology. It might seem a strange position, but it would be entirely appropriate. Close relationship between the physical and mental experience, subtle but not accidental at all. However, we believe, all the theory of dynamical systems - and for that matter all the knowledge - it may be time to re-think the creep. Now, though the idea here is simply to stimulate the reader's mind. People also provide an interesting contrast to the delicate interaction between the arrow of time and self-enrichment[4].

Now we turn to the physics discussed time as experience shows our mind. The structure of the institution with experience, 'time' mean? Is this a linear flow of the past through the present to the future? Is this a circle, forever zooming around and around? We agree that the linear flow of the 'arrow of time' is only a statistical approximation to the true nature of physical reality that is presented. At the deepest level, in physical reality, time is reversible. It introduced a measure that makes the mind 'illusion' of flash time only. Physical time as the spiral, as something that is constantly wavering back and forth, then the future is uncertain terms, how to swing and sway. But what about the time as it is to actually experience the 'stream of consciousness', the flow of events through the mind? Is this a linear movement, a movement of the past through the present to the future? What could be clearer? Many issues can be more clear, we believe. Here we establish the murky waters of phenomenology - as an individual, subjective experience. No We do not doubt the words and concepts for that matter.

If it somehow is 'damaged' by external processes, it will be able to be renovated. This enables protection to survive in difficult environments, but it can hinder the fluidity and flexibility and therefore mind needs methods and d-concretizing [4].

\section{NEW FRACTAL MECHANISMS OF PHYSICS}

In this section, we consider some new cases of fractal methods or behaviors in modern physics.

\subsection{Fractional Klein-Gordon Equation}

Partial (FDS) and fractional integral (FIS) are received considerable in recent years. In many Applications, FDS and FIS provide a more accurate model of the physical Systems under consideration. The situation becomes even more significant in quantum gravity regime 
where spacetime itself has a fractal nature. The fractionation method, ordinary space and time Differentiation operators to expand[5]

$$
\left\{\begin{array}{l}
\frac{\partial}{\partial t} \rightarrow \frac{\partial^{b}}{\partial t^{b}} \\
\frac{\partial}{\partial|x|} \rightarrow \frac{\partial^{a}}{\partial|x|^{a}}
\end{array}\right.
$$

where $\frac{\partial^{b}}{\partial t^{b}}$ is the Riemann-Liouville derivative of order $0<b \leq 1$, where the RiemannLiouvill's fractional derivative of a function $f(x)$ is defined as

$$
\frac{d^{a} f(x)}{d x^{a}}=\frac{d^{n}}{d x^{n}}\left(\frac{d^{-(n-a)} f(x)}{d x^{-(n-a)}}\right)=\frac{1}{\Gamma(n-a)} \frac{d^{n}}{d x^{n}} \int_{0}^{x} \frac{f(t) d t}{(x-t)^{1-n+a}},
$$

where $n$ is an integer that satisfies $n-1 \leq a<n$, and $\frac{\partial^{a}}{\partial|x|^{a}}$ is the Riesz derivative of order $0<a \leq 2$ (in general $a \neq b$ ). Equation (2) used for numerical solution of fractal KleinGordon equation. The choice $1<b \leq a \leq 2$ is also acceptable from this point. Since, in general $a \neq b$, differentiation with respect to space and time breaks the dimensional symmetry between these two independent observable. Restoring this symmetry requires introduction of a spatial and temporal mass scale and use of non-dimensional coordinates according to the prescription.

$$
\left\{\begin{array}{l}
\left|x^{0}\right|=|x| M_{x} \\
t^{0}=t M_{t} \\
\frac{\partial}{\partial t} \rightarrow M_{t}^{b} \frac{\partial^{b}}{\partial\left(t^{0}\right)^{b}} \\
\frac{\partial}{\partial|x|} \rightarrow M_{x}^{a} \frac{\partial^{a}}{\partial\left|x^{0}\right|^{a}}
\end{array}\right.
$$

The scalar field $\varphi\left(\left|x^{0}\right|, t^{0}\right)=\phi^{2}\left(\left|x^{0}\right|, t^{0}\right)$ may be interpreted as a probability density, where $\phi\left(\left|x^{0}\right|, t^{0}\right)$ shows the probability amplitude of locating the field at $\left|x^{0}\right|$ and $t^{0}$. Under these circumstances, one may be extrapolated to

$$
\left[\left(M_{t}^{2 b} \frac{\partial^{2 b}}{\partial\left(t^{0}\right)^{2 b}}-M_{x}^{2 a} \nabla_{0}^{2 a}\right)+m_{0}^{2}\right] \varphi=0
$$


Furthermore, also one can be cast into a symmetrical form by using the parameterization

$$
\left\{\begin{array}{l}
M_{x}^{a}=M_{t}^{b}=M \\
m_{0}^{0}=\frac{m_{0}}{M} \\
\nabla_{0}^{a}=\frac{\partial^{a}}{\partial\left|x^{0}\right|^{a}}
\end{array}\right.
$$

In these equations, $m_{0}$ is the rest mass of the particle and $M_{x}$ and $M_{t}$ are spatial and temporal mass scale. Hence, a fractal Klein-Gordon equation has the following form

$$
\left[\left(\frac{\partial^{2 b}}{\partial\left(t^{0}\right)^{2 b}}-\nabla_{0}^{2 a}\right)+\left(m_{0}^{0}\right)^{2}\right] \varphi=0 .
$$
relation

The following prescription can be used to find fractal equation from a fractal dispersion

$$
\left\{\begin{array}{l}
E^{b} \rightarrow i \frac{\partial^{b}}{\partial\left(t^{0}\right)^{b}} \\
|p|^{a} \rightarrow-i \frac{\partial^{a}}{\left.\partial\left|x^{0}\right|\right)^{a}}
\end{array}\right.
$$

From another point of view, fractal Klein-Gordon equation is given by the following fractal dispersion relation

$$
E^{2 b}=|p|^{2 a}+\left(m_{0}^{0}\right)^{2}
$$

\subsection{Fractal cosmology}

The first attempt to model the distribution of galaxies with a fractal pattern of Pietronero Luciano and his team was built in 1987, and more detailed specification of the large-scale structure of the universe emerged in the following decade, the number of larger galaxies List grew[6].

The world is homogeneous and isotropic (ie a flat distribution) on a very large scale, as in the standard Big Bang cosmology or FLRW expected, and the many interpretations of lambda cold dark matter model. The Sloan Digital Sky Survey is a scientific consensus interpretation (SDSS) showing that everything is really smooth top 100 Megaparsecs. 
Power-law spectrum Analysis another red galaxies (LRGs) in the SDSS data to calculate the fractal dimension of the galaxy distribution (scale 70-100 MPC / h) at 3, is consistent with homogeneity. The fractal dimension is 2 to almost $20 \mathrm{MPC} / \mathrm{h}$. In a 2008 article entitled 'parabolic drift towards the adoption of large-scale structures of the universe' (Physica, A, 387: 3641-3646). D. Queirs et al showed that the large scale structure of galaxies are much better drifting by a fractal dimension depending on the scale of zero to three on a scale of $55 \mathrm{MPC} / \mathrm{h}$ on the 'entropy scale diffusion equation' described. Moreover, the fractal dimension varies linearly with the logarithm scale. This means that the geometry of the distribution of galaxies is 'parabolic fractal'. Two years later, the author M. Feidt showed that the fractal dimension 2 in various studies can be explained as a problem of measurement. In 2012, Scrimgeour et al. conclusively demonstrated that the large scale structure of the universe beyond the scale of approximately $70 \mathrm{MPC} /$ hours, was close to the value found by D. Queirs [7].

In 2013, astronomers large group of quasars (LQG) is 1.6 billion light years in diameter discovered, much more than the cosmological principle, which claims that the world should allow large-scale homogeneity. In the realm of theory, fractal cosmology likely debut with Andrei Linde [7] theory 'chaotic inflationary universe itself again in eternity' (see the theory of chaotic inflation), respectively in 1986. In this theory, evolution just start scalar top of the core points that can be converted pieces of space to develop 'the bubble', making the world at large scale is a fractal. Alan Gott's 2007 article in 'eternal inflation and its consequences'[8]. This type of inflationary universe theory is still considered seriously today. Inflation, in some form is widely considered to be the best available in our cosmological model.

Since 1986, however quite a lot of different theories of cosmic exhibit fractal properties have been proposed. And while the idea of fractality Linde will likely be larger than the visible scale shows, like quantum Einstein gravity, fractals are at the opposite extreme and ultra-small territory near the scale Planck. The recent theory of quantum gravity fractal structure of space-time describe and shows dimensions of the space evolving with time [8].

In other side, French mathematician, Alain Connes, for a number of years has worked to reconcile general relativity with quantum mechanics and thus to unify the laws of physics geometry using Noncommutative. The paper also proposes an approach to quantum gravity Fractality Alexander Hellemans in August of 2006. Issue of Scientific America Connes quoted as saying that the next major step towards this goal is to 'try to understand howness of a couple with a fraction of gravity". Connes, work with physicist Carlo Rovelli shows the time of an emergency or a natural result of this formula. However, both approaches show the fractal fabric of space itself [8]. 


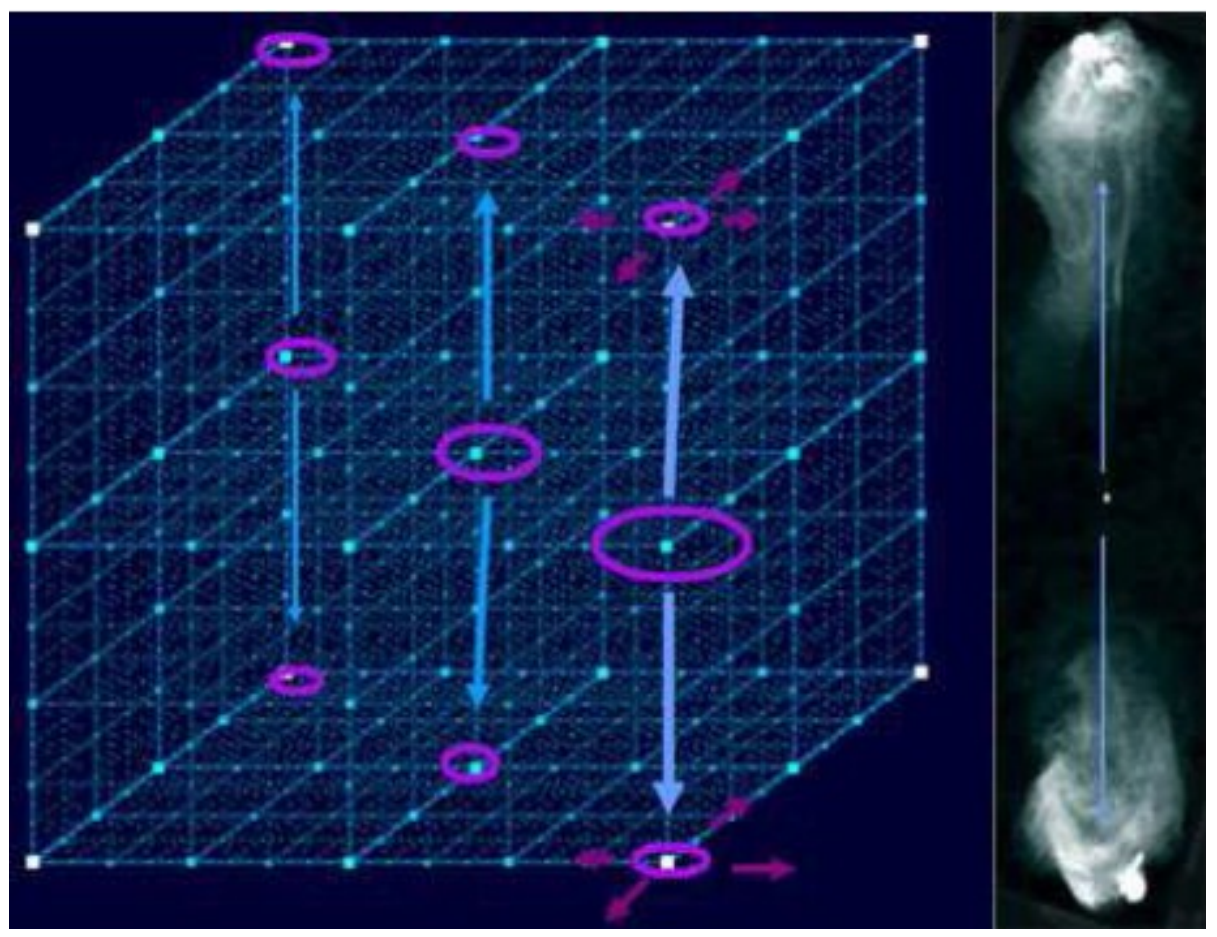

Figure 3. Suggested mechanism for the formation of new layers of galaxy clusters. A large galaxy ejects plasma spirally and axially which then spreads to sideways, Lorentz force in both cases, which accurately mimics hurricane formation.

\subsection{Fractal Meshes and Colors Interplay}

P-adic quantum mechanics is a relatively new approach to the understanding of fundamental physics. The software analyzes the P-adic quantum mechanics. P-adic numbers in an arithmetic logic was opposed by the German mathematician, Kurt Hensel, in 1899. Authors are currently studying to become an important branch of mathematics. They are sometimes used in the physical sciences, but it was not until the publication in 1987 by the Russian mathematician, Volovich that became a serious issue. Currently, there are hundreds of research papers on this subject, along with international journals as well[9]. In modern research on the subject, the Schrdinger equation is similar to the idea of exploration. Ultimately, it is the exact number of samples taken in the list.

Many studies deal with the nature of the questions that occur at the Planck length, which do not seem to be a common reality. In some ways, the test and the examiner are indistinguishable. Formalism of Hilbert space quantum mechanics and cosmology greatness present a formidable challenge. Most researchers feel that the geometry and topology of the Planck length don't need to have any relation whatever to the geometry and topology of the ordinary. According to the latter, instead of the former appearance just as the color of the flower emerges from the atom. Now many of the proposed frameworks, and the P-adic analysis is a reasonable candidate having several achievements to win[9]. Another motivation for using the P-adic analysis is differences that plague quantum field theory and the problem remains. 
There are two main methods for this issue: The first particle in a potential to consider the P-adic and the goal is to find solutions to various smooth complex wavefunctions. Here is a solution to a certain amount of familiarity of everyday life. Second particles in the potential well to consider the P-adic and the goal is to find the P-adic valued wavefunctions. In this case, the physical interpretation is more difficult. However, the mathematical most striking feature of the exhibition, so people continue to explore it.

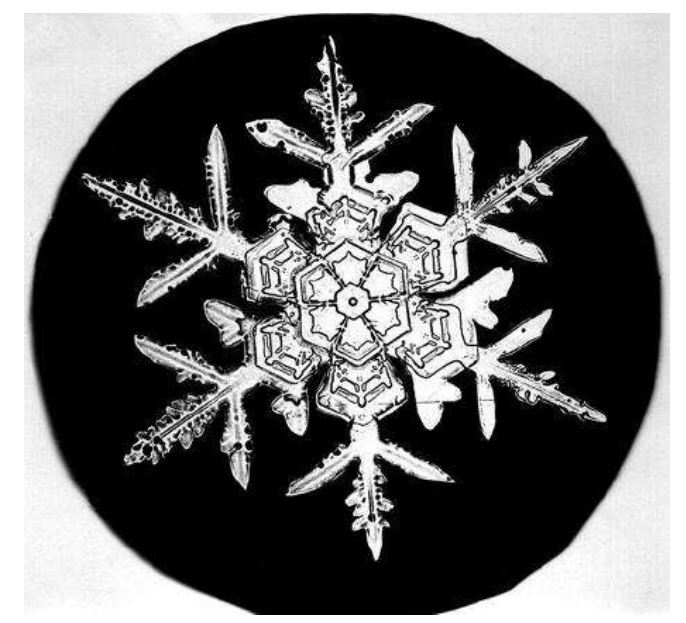

Figure 4. Symmetric patterns can emerge from seemingly unrelated ones.

\subsection{Physics of Fractal Operators}

There are many objects in the world around us that description does not fit into the rigid the Euclidean lines and surfaces. With the advent of fractals, their description has become a reality in the same place in the physics of the processes described by the average nontraditional fractal areas of mathematics. Here is just one example, the mean square distance accidentally wandering particles removed from the initial point (with equal probability in each Direction) is proportional to the time, if we're talking about continuous medium that is normal. In a Fractal, environment is not the case. Here it is clear that the particles will be randomly wandering away from the launch pad, because all parts are available for it. Mean square Fractal distance proportional to some power of the media fraction of the time, the index is the fractal dimension of the medium. This means, in particular, the publication of Environment that is fractal, continuous medium is not normal. Many obstacles (bottlenecks, Steep turns and dead ends) prevents the promotion and diffusion of the particles. Hence, Fractional rate of dependence is different[10].

Reduction in fractals emission so that it ceases to satisfy the classical Fick's law. As a result, diffusion equation, does not save the situation and tries to enter Penetration rate varies depending on the concentration. Integral differential equation contains an unusual new object a derivative (in time) of a fraction Means in relation to the average fractal dimension. Another example, is in Telecommunications, the internet, the labyrinth of the fractal structure of the portal. The purpose of these discussions is to draw attention to the strange labyrinths Fractal Theory Fractional Calculus. Moreover, it is the question of new and interesting research. 
Some developing methods for the synthesis of highly complex and artificial fractal metamaterials have been considered, for example, the fractal antenna, the fractal structure of the photon Crystal magnon, fractal modeling of impedance and fractional operators, synthesis influence, Labyrinths fractal contour blocks, etc. Fractal labyrinths are quite common in nature and technology. This should include: transportation and communication networks, systems collection and Distribution of information and resources, river systems, circulatory system, lightning Secretions and so on. The fractal nature of the geometry of the system is determined by the characteristics of the Dynamic behavior and transport properties. Fractal labyrinth connected topological structure with fractal dimension $D_{F}>1$ and the nature of the scaling of transmission channels[10].

\section{CONCLUSION}

The study of Chaos shows that simplicity and complexity can be related by considering universal properties of simple iterative processes. It was discovered that certain essential properties of chaotic systems are universal. This allows us to study a simple system and obtain conclusion for a complex system.

Fractals often occur in chaotic systems but they are not the same! Neither of them necessarily imply each other. What we would like to illustrate here is not that fractals can be used to generate beautiful pictures, but that a simple non-linear equation can be incredibly complex. However, almost everything in our world is chaotic, yet order is also everywhere. Understanding this dichotomy is a fabulous challenge. This in fact means that the most significant result of researches is that fractal calculations can actually be used to correct all the theories of modern physics.

\section{References}

[1] Falconer, Kenneth, Fractal Geometry: Mathematical Foundations and Applications. John Wiley, Ltd. xxv. ISBN 0-470-84862-6 (2003).

[2] Peters, Edgar, Chaos and order in the capital markets : a new view of cycles, prices, and market volatility. New York: Wiley. ISBN 0-471-13938-6 (1996).

[3] Losa, Gabriele A.; Nonnenmacher, Theo F., Fractals in biology and medicine. Springer. ISBN 978-3-7643-7172-2 (2005).

[4] Cramer-J-G., "An overview of the transactional interpretation of quantum mechanics", International Journal of Theoretical Physics. 27 pp. 227 (1988).

[5] S. H. Mehdipour, K. Nozari, and S. D. Sadatian, Fractals. 16 pp.33 (2008).

[6] Pietronero, L., "The Fractal Structure of the Universe: Correlations of Galaxies and Clusters". Physica A. 144 pp.257 (1987).

[7] Scrimgeour, M.; et al. "The WiggleZ Dark Energy Survey: the transition to large-scale cosmic homogeneity". Mon. Not. Roy. Astron. Soc. 425 pp.116 (2012). 
[8] Nottale, L., "The theory of Scale Relativity". Int. J. Modern Phys. A. 7 pp.4899 (1992).

[9] L. Brekke and P. G. O. Freund, P-adic numbers in physics, Phys. Rep. 233 pp.1 (1993).

[10] Metzler, R. and J. Klafter, "The random walk's guide to anomalous diffusion: A fractional dynamics approach," Phys. Rep. 339 pp.1 (2000). 